Copyright ( $[$ [2006] IEEE. Reprinted from

(Special Issue on Nonlocal, Collisionless Electron Transport in Plasmas - June 2006) .

This material is posted here with permission of the IEEE. Internal or personal use of this material is permitted. However, permission to reprint/republish this material for advertising or promotional purposes or for creating new collective works for resale or redistribution must be obtained from the IEEE by writing to pubs-permissions@ieee.org.

By choosing to view this document, you agree to all provisions of the copyright laws protecting it. 


\title{
Comparison of Two Microwave and Two Probe Methods for Measuring Plasma Density
}

Scott Knappmiller, Scott Robertson, and Zoltan Sternovsky

\begin{abstract}
Four types of electron density measurements are compared in the same device: cylindrical probe, disk probe, microwave cavity, and microwave hairpin. The measurements are made in hot-filament discharges in a soup-pot type of plasma device with and without multidipolar surface magnetic fields with densities up to $1 \times 10^{9} \mathrm{~cm}^{-3}$ and electron temperatures of 0.1-1.3 eV. The cylindrical probe and hairpin give densities that are in close agreement for all conditions. The density from the cylindrical probe, hairpin, and cavity measurements are in good agreement ( $\leq 12 \%$ difference) in plasma without magnetic containment. With magnetic containment, the different methods give greater differences in the densities $(\leq 30 \%)$, perhaps as a result of the higher fraction of energetic electrons. For all conditions, the densities from the disk probe are lower than the densities from the other methods.
\end{abstract}

Index Terms_-Plasma devices, plasma measurements, plasma sheaths

S. Knappmiller and S. Robertson are with the Center for Integrated Plasma Studies and the Department of Physics, University of Colorado, Boulder, CO 80309-0390 USA. (Email: scott.robertson@colorado.edu).

Z. Sternovsky is with the Laboratory of Atmospheric and Space Physics, University of Colorado, Boulder, CO 80309-392 USA. 


\section{INTRODUCTION}

The use of several diagnostic methods for the measurement of a single quantity often has the undesired result of creating multiple values for the quantity with differences that lie outside the expected range of error. In this work, two probe methods (disk and cylindrical) and two microwave methods of measuring electron density are compared. In the cases where the values are significantly different, possible reasons for the differences are investigated. Densities in low-temperature plasmas are most often determined from the current-voltage characteristics of Langmuir probes. Microwave methods of measuring density include the cavity method, ${ }^{1,2}$ which gives a weighted volume-averaged measurement, and the hairpin resonator method, ${ }^{3}$ which gives a localized measurement. Density measurements by these four methods are compared using a soup-pot type of plasma device, both with ${ }^{4,5}$ and without $^{6}$ surface multidipolar magnetic fields. The range of densities measured is $1 \times 10^{7} \mathrm{~cm}^{-3}$ to $1 \times 10^{9} \mathrm{~cm}^{-3}$. Without multidipolar magnetic fields, the cavity, hairpin, and cylindrical probe measurements are in good agreement and the disk probe measurement is significantly ( 40\%) lower. With multidipolar fields, the density from the cylindrical probe is slightly ( 20\%) lower than that from the microwave methods and the disk probe is again $\sim 40 \%$ lower. The probe data show that with multidipolar fields a larger fraction of the current is from suprathermal electrons and these electrons may be the origin of errors in the data analysis.

Density measurements by microwave and probe methods have previously been compared in RF and inductively-coupled discharges. ${ }^{7,8,9}$ It is likely that differences observed in the measurements are dependent upon the type of plasma device. The motivation for the present work is to have a comparison of measurement techniques that 
is valid for the types of plasmas that occur in soup-pot, double-plasma, and other types of hot-filament discharge devices. For example, the two-temperature electron distributions that are seen in double-plasma devices may affect probe measurements more than microwave measurements because a single mean electron velocity is used to convert probe currents to densities. The density of the hotter population is greatest with multidipolar magnetic fields, but in all cases is less than $10 \%$ of the density of the cold population that is confined by the plasma potential.

\section{MEASUREMENT THEORY}

\section{A. Langmuir probes}

In hot-filament discharges, the probe data often show two electron distributions with different temperatures. Our method of data analysis uses orbit-motion-limited (OML) theory ${ }^{10,11}$ to find the densities and temperatures of the two Maxwellian distributions that best fit the data. ${ }^{12}$ The ion contribution to the probe current consists of the OML ion current plus the current from charge-exchange ions created near the probe. ${ }^{13,14}$ The ion current is fit to the function described in ref. [12] and is subtracted from the net current to obtain the current from electrons alone. The electron contribution to the probe current is then decomposed into a low energy population of confined electrons (energy $<0.8 \mathrm{eV}$ ) and a high energy tail $(\sim 1-4 \mathrm{eV})$ of secondary electrons from the walls. The secondary electrons are produced from the primary electrons from the filaments impacting the chamber walls. The density of these wall secondaries is 
consistent with the value that is calculated from the current of primaries and the secondary emission coefficient of the walls. ${ }^{12}$

The two electron temperatures are determined from the two slopes of a semilogarithmic plot of the electron current. For probe voltages more negative than the wall potential, the collected current is primarily from the wall secondaries and the slope of the data indicates an effective temperature for the secondaries. The probe current from the wall secondaries is extrapolated to voltages more positive than the wall potential using OML theory for the saturation region of the current. This extrapolated current is subtracted from the total electron current to obtain the current from the confined electrons. The OML theory is fit to the current from the lower-energy population to obtain the density of the confined electrons. The total electron density is the sum of the densities of the two populations.

There is no analog of the OML theory for the disk probe current because the particle trajectories and the probe sheath are dependent upon both of the cylindrical coordinates $r$ and $z$, which complicates analysis. For positive probe voltages, there is no analytic expression for the slowly increasing electron current. For negative probe voltages, there is no theory for the ion current from charge-exchange collisions (but this has negligible impact on electron density determination). The method adopted here for the analysis of the disk probe data is the same method that is used for the cylindrical probe, with one variation. The current from the wall secondaries is assumed to be constant for probe voltages more positive than the wall potential. This assumption is consistent with disk probe theory for the saturation current. This constant current is subtracted from the probe current to obtain the current from the confined population. 


\section{B. Microwave methods}

Microwave cavity resonance methods are based upon the relative plasma permittivity, $\varepsilon / \varepsilon_{0}=1-f_{p}^{2} / f_{\text {res }}{ }^{2}$, where $\varepsilon_{0}$ is the permittivity of vacuum, $f_{p}$ is the plasma frequency and $f_{\text {res }}$ is the microwave frequency. ${ }^{1}$ If the square of the frequency of a microwave resonator in vacuum is $f_{\text {res }, 0}^{2}$, then with plasma it is $f_{\text {res }}^{2}=f_{\text {res }, 0}^{2}+f_{p}^{2}$, where $f_{p}$ is the plasma frequency. For low density plasmas satisfying $f_{p}^{2}<<f_{\text {res }, 0}^{2}$, the relationship can be linearized to obtain the electron density:

$$
n_{e} \cong \frac{8 m_{e} \varepsilon_{0} \pi^{2} f_{\text {res }, 0}}{e^{2}} \Delta f,
$$

where $\Delta f=f_{\text {res }}-f_{\text {res }, 0}$. If the plasma density is inhomogeneous, the density that is obtained is a volume average weighted by the square of the electric field of the microwaves. Insertion of probes is likely to cause the cavity modes to be different from those calculated for simple cylinders.

Localized measurements of plasma density are made by inserting a hairpin resonator consisting of a short segment of parallel wires shorted at one end and open at the other. ${ }^{3}$ This measurement of density is localized to a region of plasma with an extent determined approximately by the wire separation and length. The data are analyzed in the same way as the data from the cavity resonator. 


\section{THE APPARATUS}

\section{A. The vacuum chamber}

The plasma is generated in a double-plasma device ${ }^{6}$ from which the center grid has been removed, Fig. 1. The vacuum chamber is aluminum with a diameter of $31 \mathrm{~cm}$ and a length of $69 \mathrm{~cm}$. The aluminum walls were found to have regions of contamination that were poorly conducting, which resulted in areas of the wall being charged to negative potentials near the floating potential. ${ }^{15}$ The wall potential was made more uniform by inserting a stainless steel liner that covered both the cylindrical walls and the end walls. Data from an emissive probe showed that the wall potential with the liner was more uniform, with variations below $\sim 0.1 \mathrm{~V}$. In the device without multidipolar fields, the plasma is generated by four filaments mounted symmetrically on the end flanges. The filament bias potential is $-80 \mathrm{~V}$ and the emission ranges from $80-160 \mathrm{~mA}$. The vacuum is created by a turbomolecular pump and the base pressure is $<10^{-6}$ Torr. The working gas is argon with a fill pressure of 0.1-0.8 mTorr. Electron temperatures for the confined population are in the range $0.1-0.25 \mathrm{eV}$.

For multidipolar confinement, the device is operated with a second stainless steel liner with line cusps ${ }^{4,5}$ of nickel-plated rare-earth magnets (12.5 mm dia. x $3 \mathrm{~mm}$ ). The magnets are mounted to iron strips riveted to the liner. The mounting strips have a spacing of $75 \mathrm{~mm}$, the magnet center-to-center spacing is $18 \mathrm{~mm}$, and the field at the magnet surfaces is $0.3 \mathrm{~T}$. With this liner, the temperature of the confined population is $\sim 0.9-1.3 \mathrm{eV}$. The original configuration of four filaments, when used with the magnets, was found to give a low electron density because the filaments were within the 
multidipolar field. To obtain a higher electron density, a gap of $8 \mathrm{~cm}$ was made in a magnet row on an end flange and a single filament was installed in the region of low field. The probe configuration was altered to accommodate the single filament (Fig. 1), which gave a maximum emission of $64 \mathrm{~mA}$. In all cases the filaments were covered with grids to reduce the local perturbation to the plasma potential in the region near the filaments.

\section{B. The Langmuir probes}

The probe tips are made of stainless steel to minimize contact potential differences between the probes and the wall. The cylindrical probe is 95 microns in radius and $30 \mathrm{~mm}$ in length. The probe radius is comparable to or less than the Debye length for all conditions. The disk probe is a thin foil with a radius of $3.4 \mathrm{~mm}$. In typical conditions, the disk radius is about 30 Debye lengths. Before measurements, the probes are heated to incandescence by biasing them several hundred volts positive. After cleaning, the probe data show no hysteresis: the data obtained with the probe voltage increasing in time are identical to that obtained with the probe voltage decreasing in time. The Langmuir probes and the microwave hairpin were mounted so that they could each be placed on axis $23 \mathrm{~cm}$ from an end wall for measurements.

\section{The microwave cavities}

A small wire antenna at one end of the chamber is used to couple the microwaves into the cavity and an identical antenna at the other end is connected to a diode detector. A sweep oscillator with a range of $2-3 \mathrm{GHz}$ is used for the measurements. The 
narrowest modes in the cylindrical chamber are the $\mathrm{TE}_{0, \mathrm{~m}, \mathrm{n}}$ modes that do not require currents that cross the junctions between the cylindrical walls and lids. ${ }^{16}$ The mode used for the measurements without the multidipolar fields is at $2.39 \mathrm{GHz}$, which is nearest to the $\mathrm{TE}_{0,2,5}$ mode. A frequency of $2.75 \mathrm{GHz}$ is used with multidipolar fields which is nearest to the $\mathrm{TE}_{0,2,8}$ mode. The width of the resonant peak is approximately $9 \mathrm{MHz}$ and the center frequency is shifted by $0.4-15 \mathrm{MHz}$ with plasma. The output of the diode detector is digitized at $7 \mathrm{kHz}$ intervals during the sweep of the frequency. A cubic polynomial is fit to the peak of resonance so that the center can be found from the point of zero slope. A cubic is used rather than a quadratic because the resonances are slightly asymmetric.

The hairpin resonator for the localized density measurement is shown in Fig. 2. The hairpin is constructed from $\sim 1 \mathrm{~mm}$ dia. copper wire with a separation of $5 \mathrm{~mm}$ and a length $L=103 \mathrm{~mm}$. The hairpin may be biased relative to ground to allow investigation of the effect of the plasma sheath on the density measurement. The hairpin resonator is operated with a standing wave of $3 / 4$ wavelengths. The resonant frequency of the hairpin is approximately $2.2 \mathrm{GHz}$ and is given by $f_{\text {res }, 0}=3 c /\left(4 L \sqrt{\varepsilon_{0}}\right)$ where $c$ is the speed of light. The hairpin is operated using the same source and detector as the cavity measurement. The hairpin resonance has a width of $12 \mathrm{MHz}$ and the center of the resonance is shifted by $0.4-16 \mathrm{MHz}$ with plasma. The use of the same range of frequencies for the hairpin and the cavity allow a rapid change from one type of microwave measurement to the other. 


\section{MEASUREMENTS}

The currents from the disk probe and cylindrical probe in plasmas with and without magnetic confinement are plotted in Fig. 3. The pressure is $0.4 \mathrm{mTorr}$ and the emission currents with and without multidipolar fields are $64 \mathrm{~mA}$ and $160 \mathrm{~mA}$, respectively. The data taken without multidipolar fields show the high energy tail from the wall secondaries and clearly show a steep rise in the current at the voltage for which collection of the confined electrons begins. The data with the multidipolar fields show the same populations, but the boundary between the populations is less distinct because the temperature of the confined electrons is nearer to that of the wall secondaries.

The data from the cylindrical and disk probes were first compared to find if the temperatures were in agreement, Fig. 4. Both with and without the multidipolar fields, the temperature from the disk probe is $\sim 20 \%$ higher than that from the cylindrical probe. The difference is greatest with the multidipolar fields. The discrepancy could be due to the higher fraction of secondary electrons when multidipolar fields are used. This possibility is supported by the data with no magnetic fields which show the greatest temperature difference at low pressure. The fraction of the electrons that are wall secondaries is greatest at low pressure because ionization is reduced. The temperature is obtained from the slope of the logarithm of the current data and is thus insensitive to small changes in the probe analysis. The temperatures could not be brought into agreement by deleting, for example, a few data points on the tail of the distribution. 
The densities deduced by the four methods are compared in Fig. 5. The cylindrical probe and the hairpin resonator give densities that are in good agreement for all pressures both with and without multidipolar fields. Without magnetic fields, the average difference is $4 \%$ and with magnetic fields the average difference is $12 \%$. The disk probe, in plasmas without multidipolar fields, gives densities that are $\sim 40 \%$ lower than the densities from the cylindrical probe and the hairpin. The agreement is somewhat better with the multidipolar fields, where the disk probe gives densities lower by $~ 25 \%$. It was suggested that the secondary electrons could be responsible for the difference in temperatures measured by the two types of probes. For the differences in density measurements, however, the wall secondaries contribute less than $10 \%$ to the total electron density and it is difficult to argue that these electrons could create a $40 \%$ discrepancy in density. The electron density is determined from the saturation current measured at the plasma potential. The data in Fig. 3 and the data for all other conditions show that the disk probe collects a smaller current per unit area when at the plasma potential.

The microwave cavity measurements of density give values higher than the cylindrical probe by up to $30 \%$ with multidipolar confinement. A possible reason for the cavity measurements being greater is that the density in the chamber is not uniform. An axial scan with the cylindrical probe, Fig. 6, shows a small increase in density as the filaments are approached that could be the origin of the discrepancy. The possibility that the difference between the cavity and hairpin measurements is related to the cavity mode was investigated by making measurements with different modes, Fig. 7. The 
measurements were closely spaced in time so that the plasma conditions were nearly identical. Measurements at 2.44 GHz and 2.72 GHZ gave densities that were within a few percent of one another.

The hairpin density measurement may be affected by the sheath around the hairpin. ${ }^{9}$ This effect was investigated experimentally by varying the bias potential on the hairpin. The data, Fig. 8, show that for bias potentials that are zero or slightly negative, the measured density is only a weak function of the bias potential. For positive bias potentials, however, the density from the measurement first increased with bias voltage then decreased. The initial increase may be due to the probe being more attractive to electrons and the subsequent decrease may be caused by depletion of the plasma by electron collection. The data for negative bias voltages suggest that the sheath corrections can be omitted for probes that are grounded or slightly negative compared to the plasma potential.

\section{SUMMARY AND CONCLUSION}

Electron density measurements by cylindrical and disk probes and by the microwave cavity and microwave hairpin methods have been compared in a soup-pot type of plasma. Without magnetic field and for all plasma densities, the cylindrical probe, cavity, and hairpin methods gave measurements with an average difference of only $8 \%$. With the magnetic fields, the cavity and hairpin measurements were slightly higher than the cylindrical probe measurement. 
The density from the disk probe was always lower than that from the other methods. The area of the probe was carefully measured using microscopy and the possibility of a significant error in area was eliminated. The current collected by the disk probe per unit area when at the plasma potential was consistently less than that collected by the cylindrical probe at the same potential. The higher temperature determined from the cylindrical probe accounted for only a small part of the difference in the density measurements. No reason for the difference in the probe density (or temperature) measurements could be identified. The relatively good agreement between the measurements by the hairpin resonator and by the cylindrical probe for all conditions suggests that these are reliable methods for low-density, hot-filament discharges. 


\section{References:}

${ }^{1}$ M. A. Heald and C. B. Wharton, Plasma Diagnostics with Microwaves (Wiley, New York, 1965), Chapter 5.1.2.

${ }^{2}$ D. J. Rose and S. C. Brown, "Methods of Measuring the Properties of Ionized Gases at High Frequencies. III. Measurement of Discharge Admittance and Electron Density,” J. Appl. Phys. 23, pp. 1028-1032 (1952).

${ }^{3}$ R. L. Stenzel, “Microwave Resonator Probe for Localized Density Measurements in Weakly Magnetized Plasmas,” Rev. Sci. Instrum. 47, pp. 603-607 (1976).

${ }^{4}$ K. N. Leung, T. K. Samec and A. Lamm, “Optimization of Permanent Magnet Plasma Confinement,” Phys. Lett. 51A, pp. 490-492 (1975).

${ }^{5}$ L. N. Leung, N. Heshkowitz and K. R. MacKenzie, “Plasma Confinement by Localized Cusps,” Phys. Fluids 19, pp. 1045-1053 (1976).

${ }^{6}$ R. J. Taylor, K. R. Mackenzie, and H. Ikezi, “A Large Double Plasma Device for Plasma Beam and Wave Studies,” Rev. Sci. Instrum 43, pp. 1675-1678 (1972).

${ }^{7}$ R. M. Moroney, A. J. Lichtenberg and M. A. Lieberman, "Cavity Perturbation Measurement of Plasma Density in Complex Geometry RF Discharges,” J. Appl. Phys. 66, pp. 1618-1621 (1989).

${ }^{8}$ G. A. Hebner and I. C. Abraham, “Characterization of Electron and Negative Ion Densities in Fluorocarbon Containing Inductively Driven Plasmas,” J. Appl. Phys. 90, pp. 4929-4937 (2001).

${ }^{9}$ R.B. Piejak, V. A. Godyak, R. Garner, B. M. Alexandrovich, and N. Sternberg, “The Hairpin Resonator: A Plasma Density Measuring Technique Revisited,” J. Appl. Phys. 95, pp. 3785-3791 (2004). 
${ }^{10}$ H. Mott-Smith, Jr. and I. Langmuir, “The Theory of Collectors in Gasious Discharges,” Phys. Rev. 28, pp. 727-763 (1926).

${ }^{11}$ F. F. Chen, in Plasma Diagnostic Techniques, R. H. Huddlestone and S. L. Leonard, eds. (Academic, New York, 1965), Chap. 4.

${ }^{12}$ Z. Sternovsky and S. Robertson, "Langmuir Probe Interpretation for Plasmas with Secondary Electrons from the Wall,” Phys. Plasmas 11, pp. 3610-3615 (2004).

${ }^{13}$ Z. Sternovsky, S. Robertson, and M. Lampe, "The Contribution of Charge Exchange Ions to Cylindrical Langmuir Probe Current,” Phys. Plasmas 10, pp. 300-309 (2003).

${ }^{14}$ Z. Sternovsky, S. Robertson, and M. Lampe, "Ion Collection by Cylindrical Probes in Weakly Collisional Plasmas: Theory and Experiment,” J. Appl. Phys. 94, pp. 1374-1381 (2003).

${ }^{15}$ S. Robertson, Z. Sternovsky and B. Walch, "Reduction of Asymmetry Transport in the Annular Penning Trap,” Phys. Plasmas 11, pp. 1753-1756 (2004).

${ }^{16}$ S. Ramo, J. R. Whinnery and T. Van Duzer, Fields and Waves in Communications Electronics (Wiley, New York, 1065), p. 550. 


\section{Figure Captions}

Fig. 1. The experimental set-up without the magnets (upper) and with magnets (lower). In actual experimental set-up all three probes lie in the same plane. The microwave antennas are not shown.

Fig. 2. The hairpin resonator.

Fig. 3. Plot of the probe current per unit area as a function of probe voltage for the disk probe (dotted line) and the cylindrical probe (solid line). The unprocessed data are shown in the upper panel for no magnetic confinement and the data with ion current subtracted are shown in the middle panel without magnetic confinement and in the lower panel with magnetic confinement. The voltage scale is shifted so that the plasma potential is at zero. The filament emission is $64 \mathrm{~mA}$ with the magnetic fields and $160 \mathrm{~mA}$ without. The pressure is 0.4 mTorr.

Fig. 4. Comparison of electron temperatures obtained from the disk probe (dotted) and the cylindrical probe (solid) as a function of pressure. The upper panel is with no magnets and $160 \mathrm{~mA}$ emission and the lower panel is with magnets and $64 \mathrm{~mA}$ emission. The lines are visual guides.

Fig. 5. The densities determined from four methods: cavity (closed circles), cylindrical probe (closed squares), hairpin probe (open circle) and disk probe (open triangles). The 
discharge current is $160 \mathrm{~mA}$ without magnets, $64 \mathrm{~mA}$ with magnets, and the argon pressure is varied from $0.1-0.8 \mathrm{mTorr}$.

Fig. 6. Plasma density from the cylindrical probe data as a function of distance from the end wall. The probes were brought as near to the filaments as allowed by the design of the experiment.

Fig. 7. Plasma density as a function of pressure as determined by two different resonant modes of the cavity.

Fig. 8. Effect of bias voltage on the density determined by the hairpin. The voltage is measured relative to laboratory ground. The cylindrical probe data indicate that the plasma potential is a few tenths of a volt positive and the wall potential is $\sim 0.5 \mathrm{~V}$ negative. 

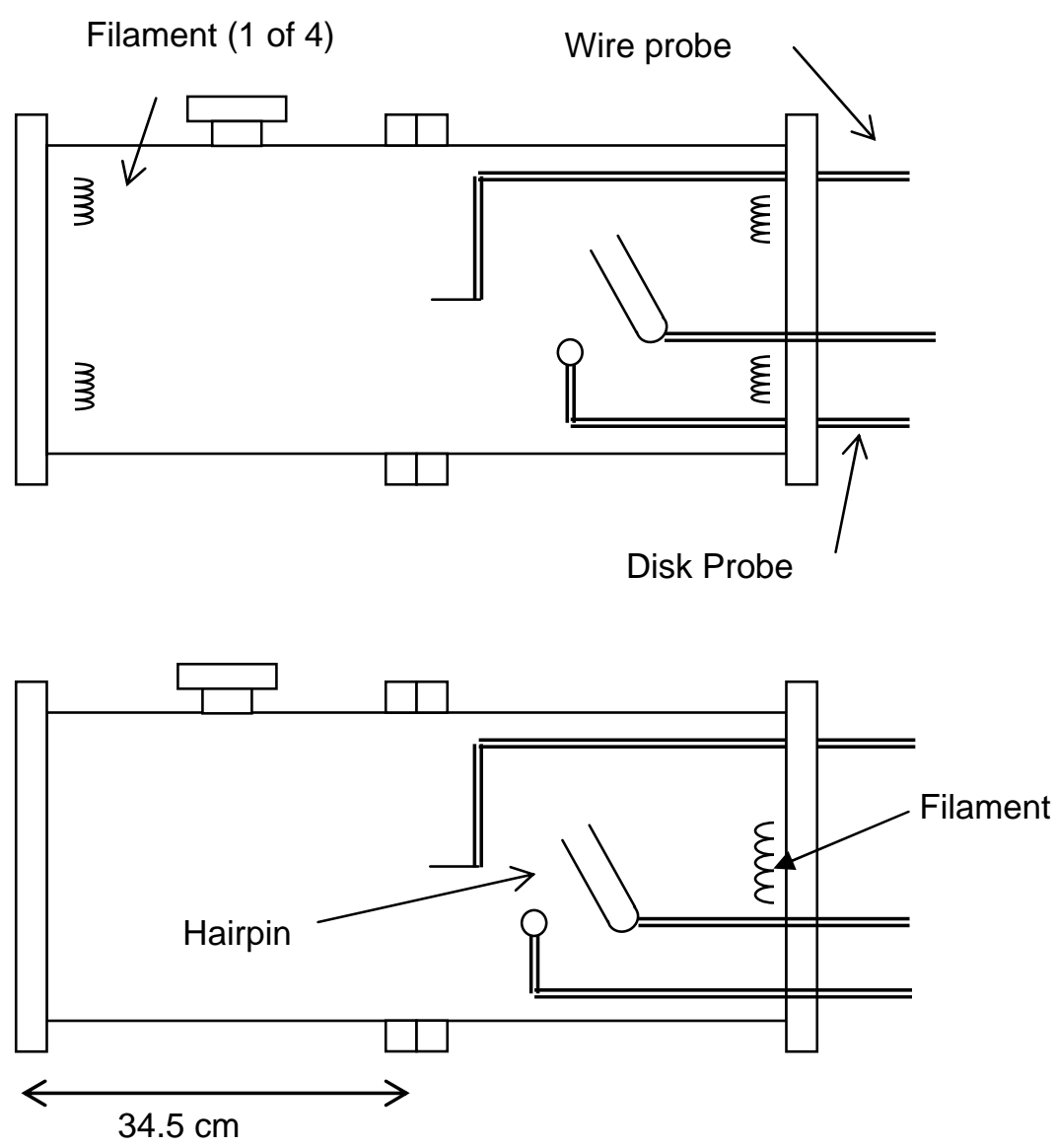

Knappmiller, Robertson, and Sternovsky, Fig. 1. 


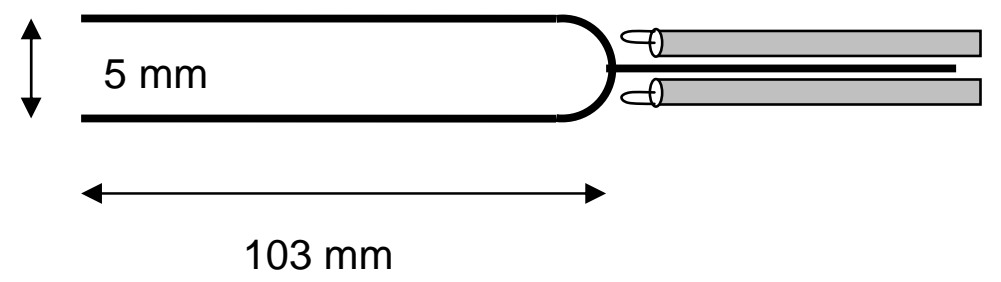

Knappmiller, Robertson, and Sternovsky, Fig. 2. 

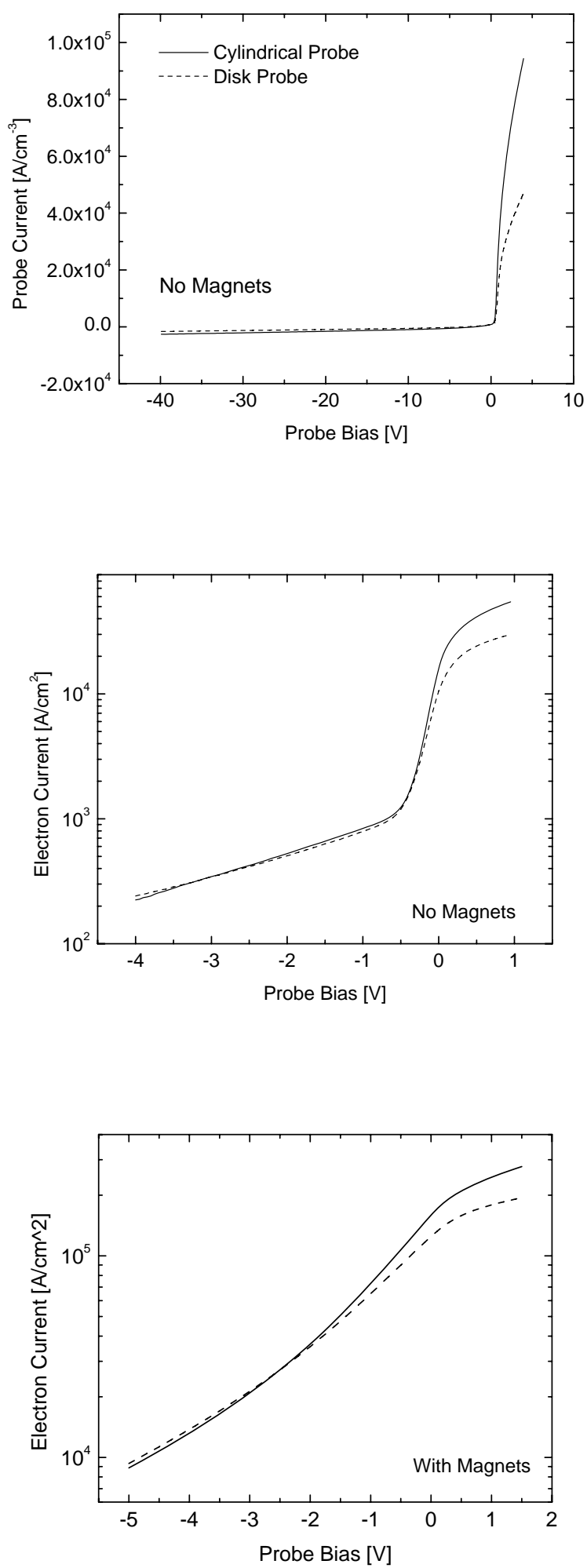

Knappmiller, Robertson, and Sternovsky, Fig. 3. 

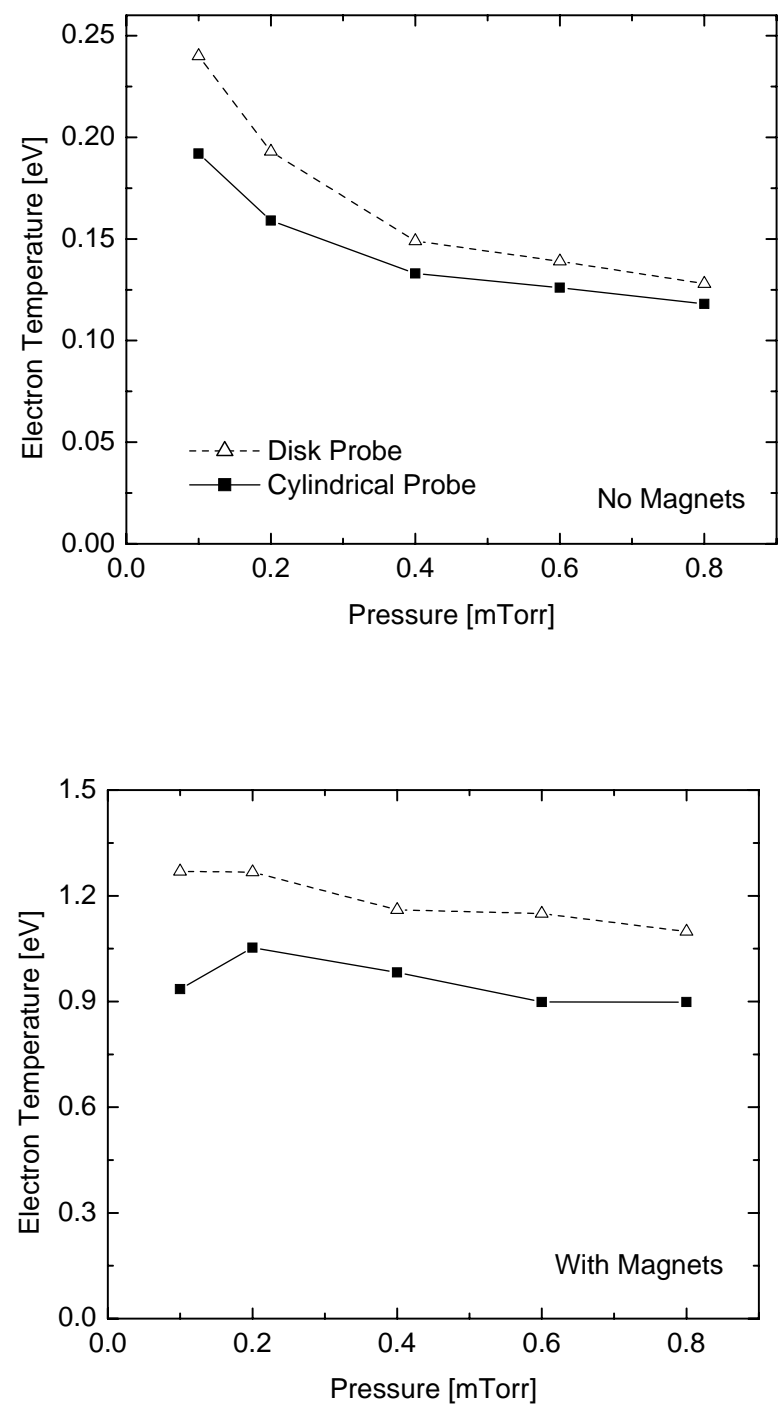

Knappmiller, Robertson, and Sternovsky, Fig. 4. 

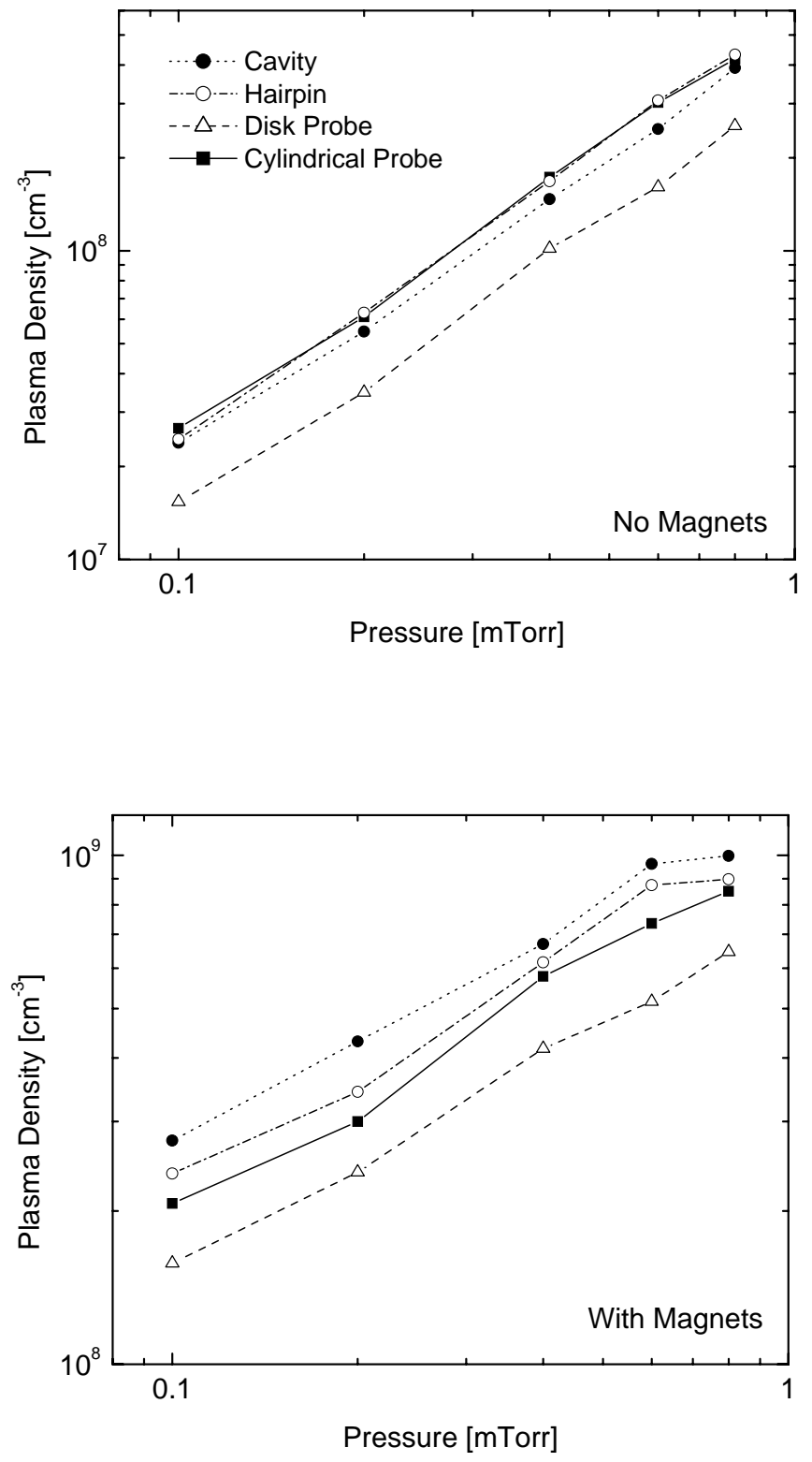

Knappmiller, Robertson, and Sternovsky, Fig. 5. 

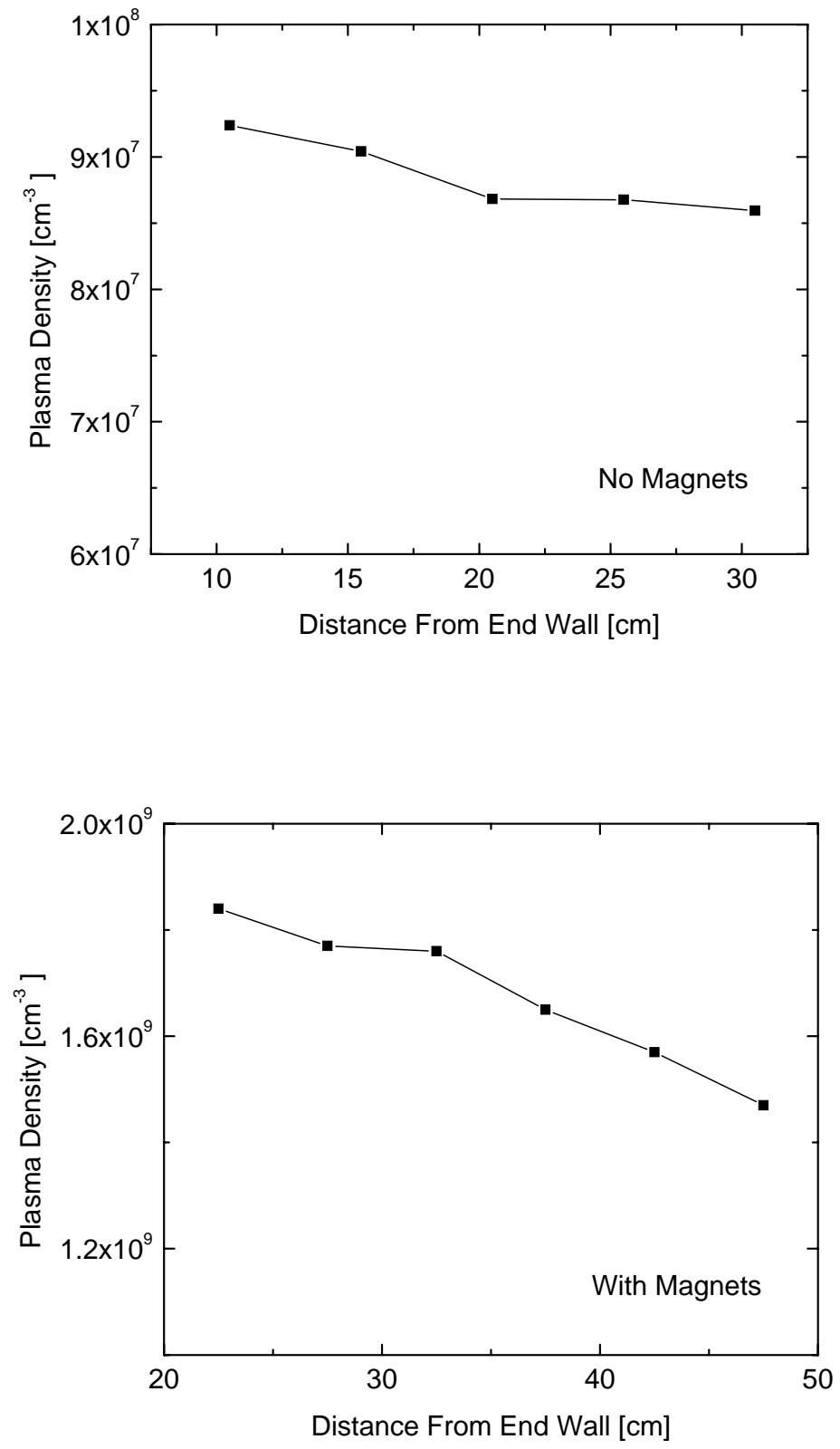

Knappmiller, Robertson, and Sternovsky, Fig. 6. 


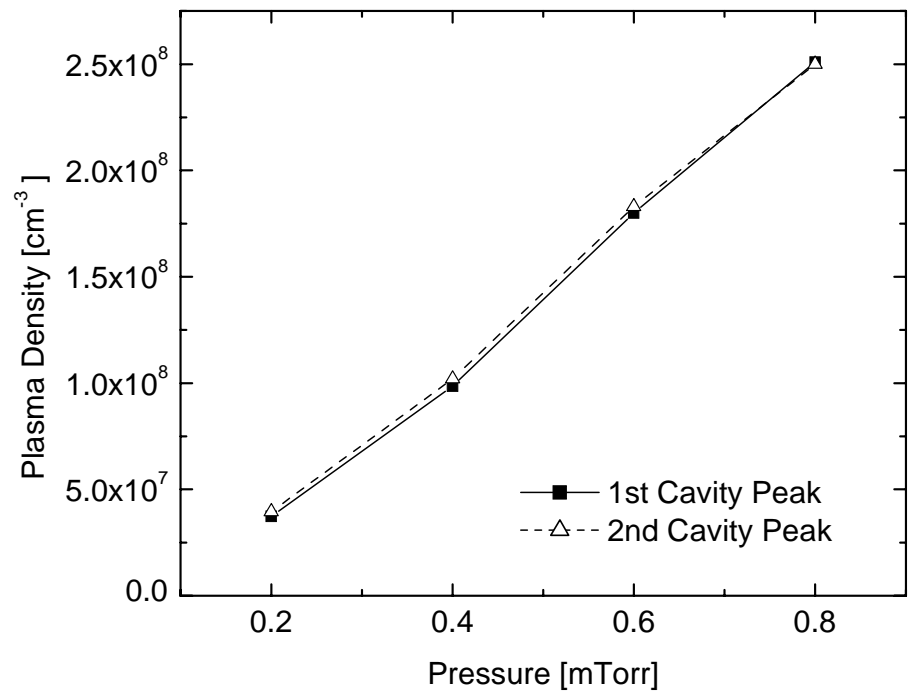

Knappmiller, Robertson, and Sternovsky, Fig. 7. 


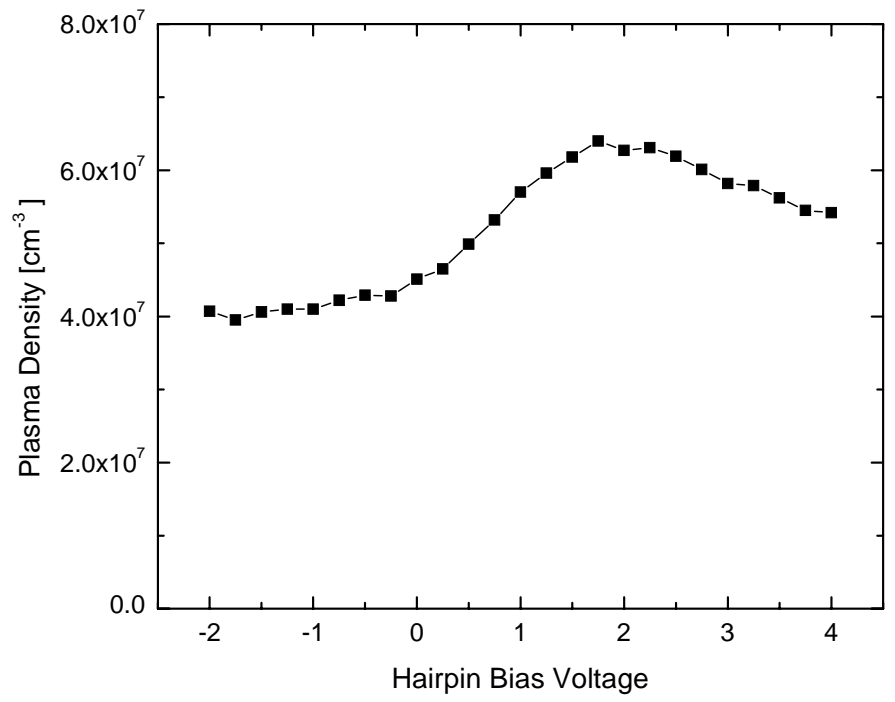

Knappmiller, Robertson, and Sternovsky, Fig. 8. 

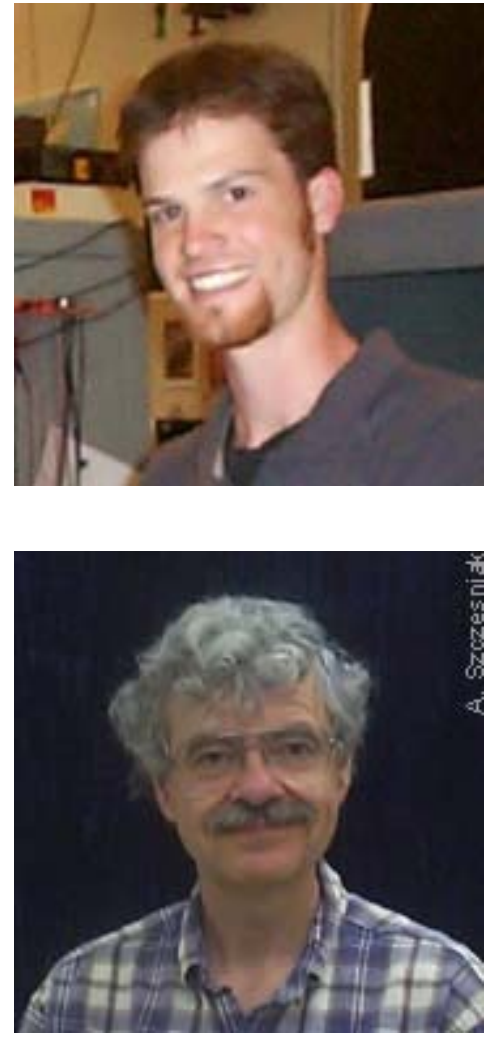

Scott Knappmiller was born in Albany, GA, in 1981. He received a B.S. degree in engineering physics and a B.A. degree in mathematics at the University of Colorado, Boulder, CO, in 2004. He is a Professional Research Assistant at the Center for Integrated Plasma Studies. His interests are in plasma diagnostic methods and instrument development for sounding rockets and spacecraft.

Scott Robertson (M'79-SM'80) was born in Washington, D.C., in 1945. He received B.S. and Ph.D. degrees in applied and engineering physics from Cornell University, Ithaca, N.Y., in 1968 and 1972, respectively. In 1973 he became a Research Associate at Columbia University in New York City where he worked on reflection and focusing of MHD shock waves. In 1975, he moved to the University of California, Irvine, CA, where he became an Associate Research Physicist and worked on collective effects in the propagation and focusing of intense pulsed electron and ion beams. In 1982, he moved to the Director of the Center for Integrated Plasma Studies. His current research interests are in dusty plasma, ionospheric plasma, and basic plasma physics.

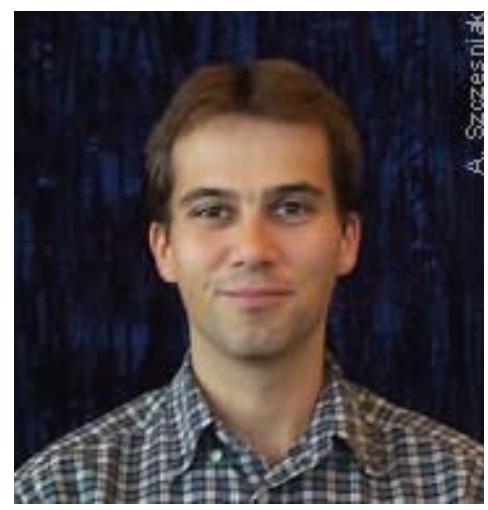

Zoltan Sternovsky was born in Nove Zamky, Slovakia, in 1974. He received M.S. and Ph.D. Degrees in physics from Charles University, Prague, in 1998 and 2001, respectively. He became a Research Assistant at the Center for Integrated Plasma Studies at the University of Colorado in 1999 and became a Research Associate in 2002. He moved to the Laboratory for Atmospheric and Space Physics in 2005. His interests are in ionospheric and space physics, basic plasma diagnostics, and in instrument development for sounding rockets and spacecraft. 